\title{
La lyophilisation des cultures de yoghourt
}

\author{
par \\ M. GAVIN \\ Institut de techniques laitières de l'Ecole Polytechnique Fédérale, \\ Zurich
}

\section{INTRODUCTION}

Les cultures de yoghourt sont composées de deux micro-organismes ayant crû en symbiose dans du lait, Streptococcus thermophilus et Lactobacillus bulgaricus (Pette et Lolkema, 1950). Ces cultures sont lyophilisées dont le but d'en améliorer la conservation et la distribution. Bien que la technique de lyophilisation ne soit plus très récente, son application aux cultures de yoghourt n'est pas fréquente et les données de littérature sont extrêmement rares. Seul le travail de Nikolov (1964) traite le problème de manière relativement complète.

La lyophilisation des cultures fraîches de yoghourt n'est pas satisfaisante (Gavin, $1968 \mathrm{~b}$ ). On obtient un produit très léger qui se dissout mal dans un liquide froid et qui n'est pas capable de donner naissance, après incubation dans du lait, à un produit ressemblant à du yoghourt. L'analyse bactériologique indique que ce lait acidifié ne contient que très peu de lactobacilles, pour la plupart filamenteux et dégénérés. C'est pourquoi nous avons tenté, au cours de ce travail, d'analyser la cause de ces résultats et de produire de meilleures cultures lyophilisées de yoghourt.

Pour y parvenir, nous avons commencé par lyophiliser des cultures pures de Lactobacillus bulgaricus et de Streptococcus thermophilus, à la suite de quoi nous nous sommes tournés vers les cultures mixtes meilleures marché à faire croître en tenant compte des observations faites sur les cultures pures.

\section{METHODES UTILISEES}

La lyophilisation des cultures de bactéries contient trois éléments principaux dont la connaissance est nécessaire pour les réalisations pratiques. Le premier comprend les conditions de nutrition et 
de croissance de la culture à lyophiliser. Le second concerne le milieu dans lequel les bactéries se trouvent au moment de la lyophilisation. Le troisième réunit les facteurs physiques réglant la sublimation et la dessiccation secondaire. Alors que les deux premiers éléments sont spécifiques à la lyophilisation des bactéries, le troisième est important pour tous les problèmes de lyophilisation.

\section{a) Cultures et méthodes de cultures}

Nous avons fait croître en cultures mixtes et en cultures pures un certain nombre de cultures de yoghourt prélevées dans le commerce. Nous avons inoculé les cultures mixtes à du lait écrémé stérile reconstitué de manière standard $(10$ p. 100$)$ à partir de poudre de lait écrémé provenant d'une seule fabrication, et les cultures pures dans les deux milieux décrits ci-dessous:

\section{Streptococcus thermophilus:}

- Trypticase BBL : $1 \mathrm{~g}$

- Hydrolysat de caséine NBC : $1 \mathrm{~g}$

- Lait écrémé standard ou sérum lactique déprotéiné : $1000 \mathrm{ml}$

$-\mathrm{pH}: 6.60$;

\section{Lactobacillus bulgaricus :}

- Lait peptonisé Difco : $15 \mathrm{~g}$

- Lait écrémé standard ou sérum lactique déprotéiné : $1000 \mathrm{ml}$ - $\mathrm{pH}: 5.80$.

Le sérum lactique déprotéiné, nécessaire à la confection de milieux transparents, a été produit par chauffage du petit lait de fromagerie acidifié à l'acide acétique au point isoélectrique. Les milieux transparents nous ont permis d'établir, à l'aide d'un spectrophotomètre et d'une courbe étalon mesurée gravimétriquement, les courbes de croissance des cultures. Ne coagulant pas comme le lait, ils nous ont permis également d'en séparer les bactéries par centrifugation et de resuspendre ces dernières dans un nouveau milieu de lyophilisation.

\section{b) Milieu de lyophilisation}

Pour les essais analytiques, les cultures ont été séparées de leur milieu de croissance par centrifugation et resuspendues dans un milieu de lyophilisation constitué de lait écrémé additionné de sucre et de glutamate de sodium (Fry, 1951, Fry et Greaves, 1951, Cho et Obayashi, 1956, Cho et al., 1956, Scott, 1958). Pour les essais de production (cultures lyophilisées avec le milieu de culture), nous nous sommes contentés d'ajouter aux cultures du sucre et du glutamate de sodium. 


\section{c) Méthode de lyophilisation}

Nous avons fait tous nos essais à l'aide d'un lyophilisateur Secfroid (Lyoboy) d'une capacité de deux litres. La chambre de dessiccation permettant également de congeler les cultures, nous les avons réparties $(1 \mathrm{ml})$ dans des tubes à pastilles (fond plat, hauteur $100 \mathrm{~mm}$, diamètre $20 \mathrm{~mm}$ ) en verre brun que nous avons disposés au fond de la cuve prérefroidie à $-40^{\circ} \mathrm{C}$. La vitesse de congélation était de l'ordre de $1^{\circ} \mathrm{C} / \mathrm{s}$. La sublimation qui durait quelques deux heures (cuve chauffée à $30^{\circ} \mathrm{C}$ ) ainsi que la dessiccation secondaire (également $30^{\circ} \mathrm{C}$ ) étaient surveillées à l'aide d'un thermomètre thermocouple.

Nous avons, dans des essais faisant varier la vitesse de congélation (de $10^{\circ} \mathrm{C} / \mathrm{s}$ à $1{ }^{\circ} \mathrm{C} / \mathrm{mn}$ ) et de sublimation (de $1 \mathrm{~h}$ à $5 \mathrm{~h}$ ), trouvé que les facteurs physiques n'avaient qu'une faible influence sur le taux de survie des bactéries. Cette constatation nous a permis, pour les essais de production, d'adopter comme récipient des flacons à sérum recevant une plus grande quantité de culture, congelée en coquille dans un bain d'acétone et de glace carbonique.

\section{d) Conditionnement des cultures}

Après avoir vérifié au manomètre que la dessiccation secondaire était achevée, nous avons cassé le vide avec de l'azote et fermé les récipients sous ce même gaz. S'il s'agissait de cultures lyophilisées dans des flacons à sérum, nous avons broyé la croûte sèche dans une atmosphère aseptique déshydratée et dosé la poudre dans des tubes à pastilles que nous avons également fermés sous azote. Ces tubes, destinés au contrôle d'activité, ont été stockés à diverses températures à l'abri de la lumière.

\section{e) Contrôle des cultures}

Après lyophilisation, nous avons mesuré chez les cultures pures comme chez les cultures mixtes, le pouvoir d'acidification en les inoculant ( 5 p. 100) au même milieu que celui qui a permis leur croissance et les incubant à $42^{\circ} \mathrm{C}$. En comparant ce pouvoir d'acidification à celui de la culture fraîche inoculée au même milieu, il a été possible de tirer certaines conclusions sur la vitalité de la culture après lyophilisation. En fait, pour les cultures pures, nous avons établi un système permettant d'estimer, sur la base de cette comparaison, le taux de survie de la culture. Nous le décrivons en détail (taux comparé de survie) dans le travail cité plus haut (Gavin, 1968 b).

Pour le contrôle des cultures mixtes, la comparaison des courbes d'acidification ne nous permettait pas de déterminer les taux respectifs de survie des deux symbiotes. C'est pourquoi nous avons introduit la notion de caractéristiques d'acidification composées des 
temps qu'il fallait pour parvenir aux pH de 5.00, 4.30 et 3.90 ainsi que de la proportion de streptocoques et de lactobacilles à ces $\mathrm{pH}$. De plus, chaque fois que cela était possible, nous avons procédé au contrôle organoleptique des yoghourts faits avec ces cultures.

\section{LA LYOPHILISATION DES CULTURES PURES}

Ayant isolé plusieurs souches de Lactobacillus bulgaricus et de Streptococcus thermophilus selon les méthodes usuelles d'isolation, nous les avons resuspendues dans un milieu protecteur et les avons lyophilisées à plusieurs moments de leur croissance dans le but de déterminer les taux respectifs de survie ainsi que l'influence de l'âge de la culture sur son taux de survie à la lyophilisation. Les tableaux $1 \mathrm{a}$ et $\mathrm{b}$ rapportent les résultats des contrôles effectués sur ces cultures.

TABLEAU $1 \mathrm{a}$ : Contrôle par acidification et taux comparés de survie de deux cultures lyophilisées de Streptococcus thermophilus (a) et de Lactobacillus bulgaricus (b) ayant crû dans les milieux transparents décrits ci-dessus. Les cultures ont été centrifugées et resuspendues dans du lait écrémé avant la lyophilisation.

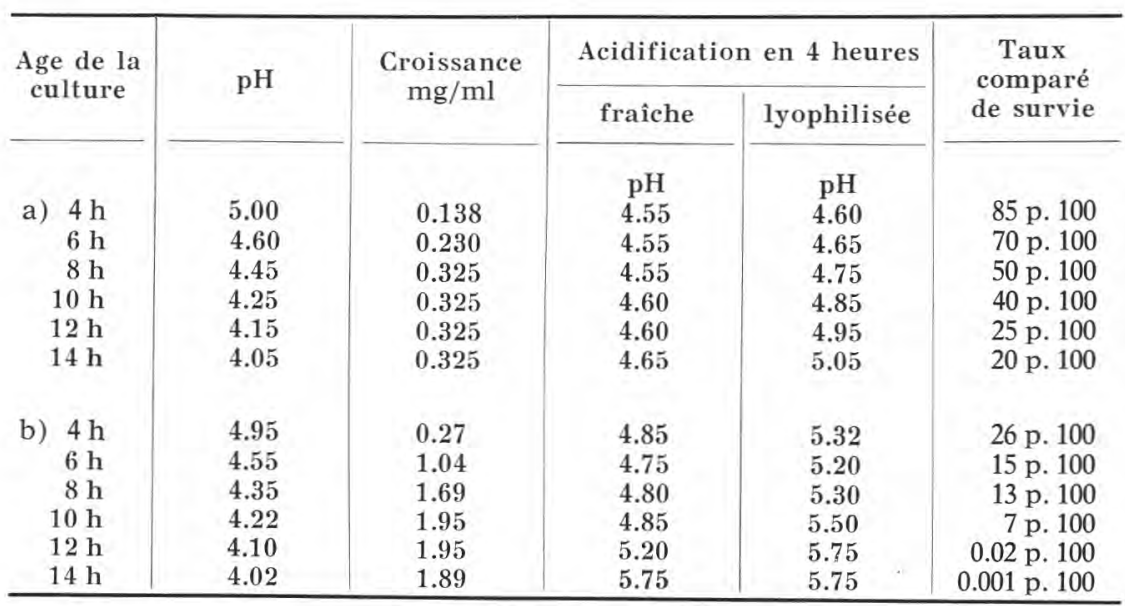

Ces tableaux nous permettent d'apprécier l'influence de l'espèce, de l'âge de la culture et du milieu de croissance ou de lyophilisation sur la sensibilité des cultures à la dessiccation. La meilleure résistance de Streptococcus thermophilus à la lyophilisation est la cause du déséquilibre observé plus haut et apparaît nettement dans les deux cas. 
TABLEAU $1 \quad b$ : Contrôle par acidification et taux comparés de survie d'une culture de Streptococcus thermophilus (a) et de Lactobacillus bulgaricus (b) ayant crû dans les milieux à base de lait écrémé décrits ci-dessus. Ces cultures ont été lyophilisées avec leur milieu de culture.

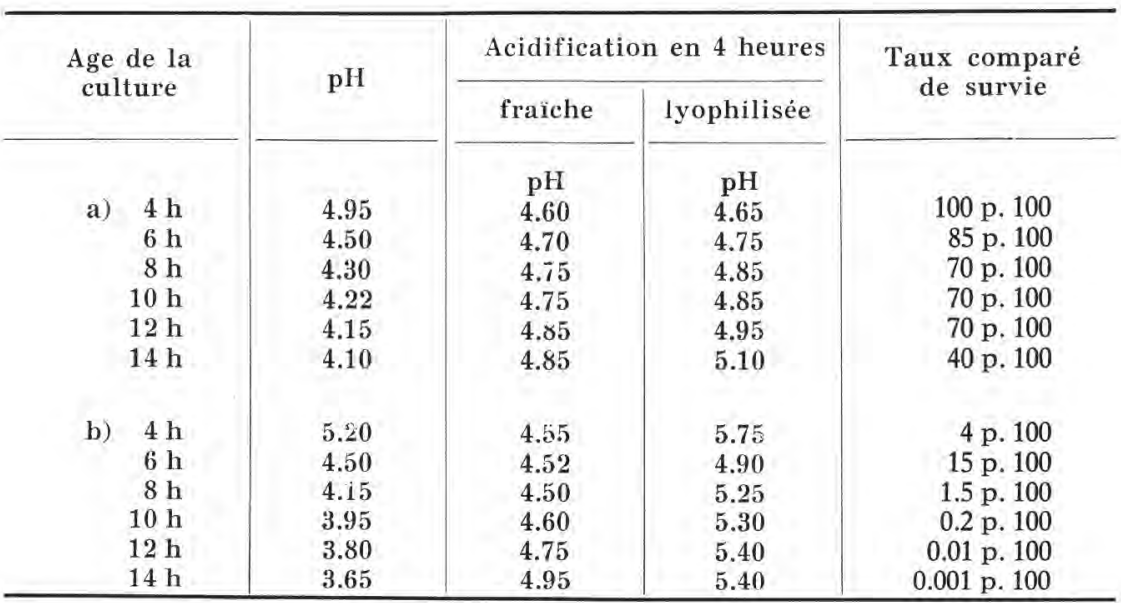

L'avantage qu'il y a de lyophiliser de jeunes cultures (au cours de la phase logarithmique de croissance) ainsi que l'influence des milieux de culture et de lyophilisation est également facile à distinguer. Bien qu'il ne soit pas possible de dire, à la suite de cet essai, quelle est la part du milieu de culture et celle du milieu de lyophilisation, nous constatons au tableau 1a que les cultures ayant crû dans les milieux à base de lait écrémé et n'ayant pas pu être séparées de leur milieu de croissance montrent des chiffres plus extrêmes que celles qui ont été centrifugées et resuspendues dans du lait écrémé. La meilleure survie des bâtonnets lorsqu'ils sont séparés de leur milieu de culture ainsi que la possibilité d'exclure les effets incontrôlables que le milieu de croissance, apporté avec l'inoculum, peut avoir sur l'acidification de contrôle nous ont conduit, à l'essai suivant, à adopter une méthode nous permettant, pour les cultures mixtes également, de séparer les bactéries de leur milieu de croissance.

\section{LA LYOPHILISATION DES CULTURES MIXTES SEPAREES DE LEUR MILIEU DE CROISSANCE}

Deux cultures mixtes de yoghourt ont été cultivées à $42^{\circ} \mathrm{C}$ dans du lait écrémé stérile additionné d'une faible dose de présure. Vers $\mathrm{pH}=5.50$, nous avons provoqué la synérèse en remuant le caillé et, à $\mathrm{pH}=4.35$, nous avons prélevé le sérum que nous avons immédiatement centrifugé. Ce mode de culture est appelé plus loin : culture 
de lait emprésuré. Ayant resuspendu le sédiment dans le milieu de lyophilisation (lait écrémé stérile +8 p. 100 de saccharose +1.5 p. 100 de glutamate de sodium), nous l'avons lyophilisé, conditionné et soumis au contrôle d'acidification.

Nous rapportons les résultats de ce contrôle au tableau 2.

TABLEAU 2. - Contrôle d'acidification de deux cultures mixtes de yoghourt lyophilisées après séparation du milieu de croissance et resuspension dans un milieu de lyophilisation. Inoculation ( 5 p. 100) à du lait bouilli et incubation à $42^{\circ} \mathrm{C}$.

\begin{tabular}{|c|c|c|c|c|c|c|}
\hline \multirow{3}{*}{ Culture } & \multirow{3}{*}{$\begin{array}{c}\text { voir } \\
\text { ci-dessous }\end{array}$} & \multicolumn{5}{|c|}{ Contrôle } \\
\hline & & Avan & Après & \multicolumn{2}{|c|}{ Conservation à $45^{\circ} \mathrm{C}$} & \\
\hline & & \multicolumn{2}{|c|}{ lyophilisation } & 7 jours & 14 jours & 28 jours \\
\hline \multirow[t]{4}{*}{ A } & I & $3^{20}$ & $3^{30}$ & $4^{30}$ & $6 \mathrm{~h}$ & $8 \mathrm{~h}$ \\
\hline & II & $4^{30}$ & $5^{55}$ & $6^{45}$ & $9^{30}$ & $12^{30}$ \\
\hline & III & $1^{10}$ & $2^{25}$ & $2^{15}$ & $3^{30}$ & $4^{30}$ \\
\hline & IV & 1.0 & 2.0 & 0.35 & 0.50 & 1.0 \\
\hline \multirow[t]{4}{*}{ B } & I & $3^{40}$ & $3^{40}$ & $4^{35}$ & $6^{20}$ & $9 \mathrm{~h}$ \\
\hline & II & $5^{10}$ & $6^{15}$ & $6^{45}$ & $9^{30}$ & $12^{30}$ \\
\hline & III & $1^{30}$ & $2^{35}$ & $2^{10}$ & $3^{10}$ & $3^{30}$ \\
\hline & IV & 1.0 & 1.0 & 0.50 & 0.50 & 0.50 \\
\hline
\end{tabular}

I : temps en heures pour atteindre le $\mathrm{pH}$ de $\mathbf{5 . 0 0}$

II : temps en heures pour atteindre le $\mathrm{pH}$ de 4.30

III $: \Delta t:$ II - I

IV : Indice lactobacilles/streptocoques à $\mathrm{pH}: 4.30$

Bien que les cultures lyophilisées aient une acidification un peu plus lente que les cultures fraîches, la séparation du milieu de croissance ainsi que la resuspension des bactéries dans un milieu de lyophilisation a pour effet un rétablissement complet de l'équilibre, équilibre qui résiste même à des conditions de stockage très rigoureuses.

Ce traitement améliore les qualités organoleptiques du yoghourt de cultures lyophilisées. Celui-ci est bon lorsqu'il est préparé avec des cultures fraîchement lyophilisées. Si les cultures ont été stockées quinze jours et plus à $45^{\circ} \mathrm{C}$, le yoghourt n'est plus aussi bon, mais en deuxième passage déjà, il redevient parfaitement normal.

La simple élimination de substances apportées avec le milieu de croissance a des conséquences très grandes sur la qualité des cultures lyophilisées de yoghourt. Le tableau 3 nous montre que ces substances sont très probablement les peptides et acides aminés 
hydrolysés par Lactobacillus bulgaricus dans le lait et qui représentent l'aliment principal de Streptococcus thermophilus.

Pour le démontrer, nous avons inoculé 2.5 p. 100 de culture lyophilisées après séparation du milieu de croissance à du lait bouilli et à du lait qui avait reçu de faibles quantités de protéines lactiques hydrolysées ( 0.1 p. 100 de trypticase et de caséine hydrolysée). Nous avons nommé activateurs ces substances capables de stimuler la croissance de Streptococcus thermophilus.

TABLEAU 3. - Influence des activateurs de Streptococcus thermophilus sur la croissance des trois cultures de yoghourt lyophilisées après séparation du milieu de croissance. Taux d'inoculation : 2.5 p. 100, température d'incubation : $42^{\circ} \mathrm{C}$.

\begin{tabular}{|c|c|c|c|c|c|c|}
\hline \multirow{4}{*}{$\begin{array}{c}\text { Voir } \\
\text { ci-des- } \\
\text { sous }\end{array}$} & \multicolumn{6}{|c|}{ Milieu de croissance } \\
\hline & \multirow{2}{*}{\multicolumn{3}{|c|}{$\begin{array}{c}\text { Lait bouilli pur } \\
\text { culture }\end{array}$}} & \multirow{2}{*}{\multicolumn{3}{|c|}{$\begin{array}{c}\text { Lait avec activateurs } \\
\text { culture }\end{array}$}} \\
\hline & & & & & & \\
\hline & A & B & C & A & B & C \\
\hline I & $6^{35}$ & $6^{40}$ & $6^{15}$ & $4^{30}$ & $4^{05}$ & $4^{20}$ \\
\hline II & $8^{30}$ & $8^{10}$ & $7^{50}$ & $5^{50}$ & $5^{25}$ & $5^{40}$ \\
\hline III & $1^{55}$ & $1^{30}$ & $1^{35}$ & $1^{20}$ & $1^{20}$ & $1^{20}$ \\
\hline IV & 0.80 & 1.00 & 0.40 & 0.025 & 0.12 & 0.05 \\
\hline
\end{tabular}

I : temps en heures pour atteindre le $\mathrm{pH}$ de 5.00

II : temps en heures pour atteindre le $\mathrm{pH}$ de 4.30

III : $\Delta \mathrm{t}:$ II - I

IV : indice lactobacilles/streptocoques à $\mathrm{pH}: 4.30$

Les lactobacilles, affaiblis par la lyophilisation plus que les streptocoques, n'ont pratiquement pas le temps de se développer dans le temps qui suffit à faire du yoghourt. L'acidification est donc principalement due aux streptocoques. La séparation des produits de protéolyse empêche la croissance prématurée des streptocoques, croissance qui devient alors entièrement dépendante de celle de Lactobacillus bulgaricus.

\section{LYOPHILISATION DES CULTURES MIXTES DE YOGHOURT EN PRESENCE DE LEUR MILIEU DE CROISSANCE}

Si la séparation des bactéries de leur milieu de croissance permet de produire des cultures équilibrées, elle introduit une opéra 
tion onéreuse qui est la centrifugation du sérum. De plus, l'emploi successif de deux milieux pour la même culture augmente encore les frais de production. Il serait donc préférable, pour la rentabilité de l'entreprise, de rendre possible la lyophilisation des bactéries avec leur milieu de croissance.

En relation avec le premier des éléments de la lyophilisation décrits au début du paragraphe 2, nous avons pensé qu'il fallait, par des méthodes de culture, chercher à élever la résistance de Lactobacillus bulgaricus seul au sein de la culture mixte.

Nous avons eu, à plusieurs reprises, l'occasion de constater (Gavin, 1968 b) que des conditions de culture favorables à un taux de survie à la lyophilisation élevé se manifestaient chez Lactobacillus bulgaricus par des bâtonnets particulièrement courts. Or, une croissance rapide dans un milieu nutritif très riche conduisait à des bâtonnets également très courts ayant un taux de survie élevé à la lyophilisation. C'est alors que nous avons essayé de cultiver les cuitures de yoghourt non plus dans du lait simple, mais dans du lait écrémé concentré à 20 p. 100 de matières sèches. Ce mode de culture est appelé plus loin : culture de lait concentré. Les résultats furent positifs. Le lait écrémé concentré offrait aux cultures de yoghourt d'excellentes conditions de crosssance. La teneur en bactéries était plus que doublée. Non seulement la proportion de bâtonnets était légèrement accrue, mais encore, ils avaient une longueur comprise entre 3 et $5 \mu$, ce qui est court pour Lactobacillus bulgaricus.

Nous rapportons au tableau 4 les résultats d'un essai de lyophiJisation de deux cultures ayant crû̀ dans du lait concentré et ayant été Iyophilisées avec le milieu de culture après adjonction de 1.5 p. 100 de glutamate de sodium. L'adjonction de sucre ne s'est pas avérée nécessaire à cause du bon pouvoir protecteur du lait concentré.

Les deux cultures lyophilisées après croissance dans du lait concentré sont restées équilibrées et ont donné un yoghourt de bonne qualité. Nous pensons cependant que la résistance accrue des bâtonnets ne suffit pas à expliquer ce résultat. A cette résistance, il faut ajouter l'accroissement numérique des lactobacilles ainsi que le très bon pouvoir protecteur du lait concentré. Il est par ailleurs possible que la température choisie pour l'épreuve accélérée de conservation $\left(45^{\circ} \mathrm{C}\right)$ soit trop haute et qu'elle détruise les acides aminés libres autant que les bactéries.

\section{COMPARAISON ENTRE CULTURES SEPAREES DE LEUR MILIEU DE CROISSANCE ET CULTURES DE LAIT CONCENTRE}

Nous possédons maintenant deux méthodes nous permettant, par des moyens fort différents, de produire des cultures lyophilisées actives et équilibrées. Pour permettre une meilleure appréciation de 
TABLEAU 4. - Contrôle de deux cultures mixtes de yoghourt ayant crû dans du lait écrémé concentré (20 p. $100 \mathrm{MS})$ et lyophilisées avec le milieu de croissance. Inoculation : 5 p. 100 du lait bouilli et incubation à $42^{\circ} \mathrm{C}$.

\begin{tabular}{|c|c|c|c|c|c|c|}
\hline \multirow{3}{*}{ Culture } & \multirow{3}{*}{$\begin{array}{l}\text { voir ci-des- } \\
\quad \text { sous }\end{array}$} & \multicolumn{5}{|c|}{ Contrôle } \\
\hline & & Avant & Après & \multicolumn{3}{|c|}{ Conservation à $45^{\circ} \mathrm{C}$} \\
\hline & & \multicolumn{2}{|c|}{ lyophilisation } & 7 jours & 14 jours & 28 jours \\
\hline \multirow[t]{4}{*}{$\mathbf{A}$} & I & $2 \mathrm{~h}$ & $2^{25}$ & $4 \mathrm{~h}$ & $5^{25}$ & $6^{35}$ \\
\hline & II & $3^{15}$ & $4^{40}$ & $7 \mathrm{~h}$ & $9 \mathrm{~h}$ & $12 \mathrm{~h}$ \\
\hline & III & $1^{15}$ & & $3 \mathrm{~h}$ & $3^{35}$ & \\
\hline & IV & 1.0 & 4.0 & 0.35 & 0.50 & 1.0 \\
\hline \multirow[t]{4}{*}{ B } & I & $2^{05}$ & $2^{10}$ & $4^{15}$ & $5^{30}$ & $7^{50}$ \\
\hline & II & $3^{25}$ & $4 \mathrm{~h}$ & $6^{45}$ & $8^{30}$ & $11^{15}$ \\
\hline & III & $1^{20}$ & $1^{50}$ & $2^{30}$ & $3 \mathrm{~h}$ & $3^{25}$ \\
\hline & IV & 1.0 & 2.0 & 1.0 & 1.0 & 1.0 \\
\hline
\end{tabular}

I : temps en heures pour atteindre le $\mathrm{pH}$ de 5.00

II : temps en heures pour atteindre le $\mathrm{pH}$ de 4.30

III : $\Delta \mathrm{t}:$ II - I

IV : indice lactobacilles/streptocoques à $\mathrm{pH}: 4.30$

ces deux méthodes, nous avons procédé à un essai comparatif de plus grande envergure sur trois bonnes cultures de yoghourt ayant crû, d'une part, dans du lait écrémé emprésuré et, d'autre part, dans du lait écrémé concentré. Ces cultures avaient, après croissance, les caractéristiques suivantes:

\begin{tabular}{c|c|c|c|c}
\hline \multirow{2}{*}{ Culture } & \multicolumn{2}{|c|}{ Lait concentré } & \multicolumn{2}{c}{ Lait emprésuré } \\
\cline { 1 - 1 } $\mathrm{E}$ & $\mathrm{pH}$ & $\mathrm{Lb} / \mathrm{Sc}$ & $\mathrm{pH}$ & $\mathrm{Lb} / \mathrm{Sc}$ \\
\cline { 1 - 2 } 4 & 4.40 & 1.0 & 4.10 & 2.0 \\
$\mathrm{~N}$ & 4.50 & 0.25 & 4.05 & 1.0 \\
$\mathrm{U}$ & 4.40 & 1.0 & 4.05 & 2.0 \\
\hline
\end{tabular}

Alors que les cultures de lait emprésuré étaient centrifugées et resuspendues dans du lait écrémé contenant 10 p. 100 de saccharose et 1.5 p. 100 de glutamate de sodium, celles de lait concentré ne reçurent avant la lyophilisation, que le glutamate de sodium. Les tableaux suivants (5 et 6 ) rapportent les résultats des contrôles d'acidification. 
TABLEAU 5 : Contrôle d'acidification de trois cultures de yoghourt lyophilisées après séparation de leur milieu de croissance et resuspension dans un milieu de lyophilisation. Influence de la température de stockage et de la dose d'inoculation. Contrôle par inoculation à du lait complet bouilli et homogénéisé et incubation à $42^{\circ} \mathrm{C}$.

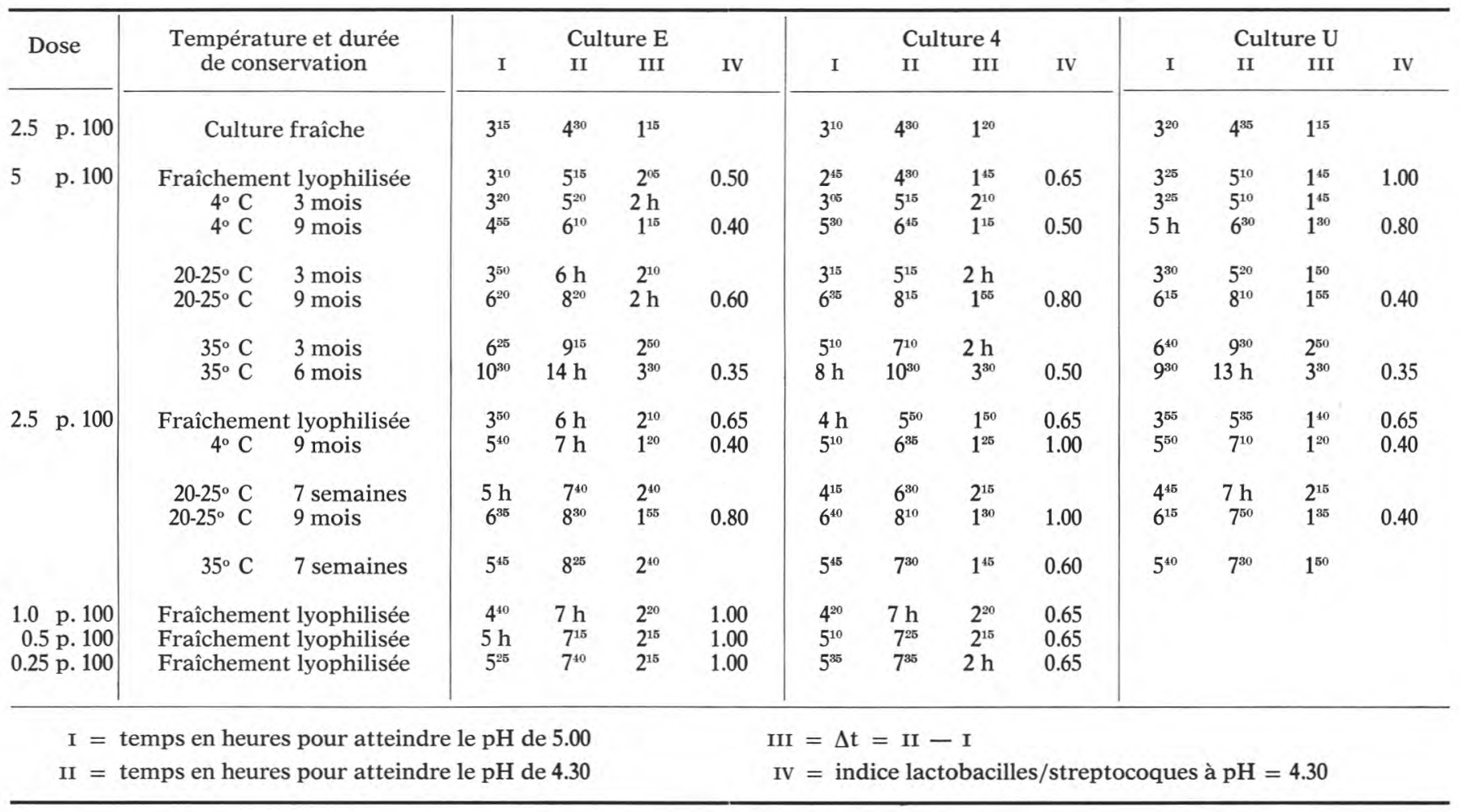


TABLEAU 6. - Contrôle d'acidification de trois cultures lyophilisées de yoghourt ayant crû dans du lait écrémé concentré (20 p. 100 MS). Influence de la température de stockage et de la dose d'inoculation. Contrôle par inoculation à du lait bouilli homogénéisé et incubation à $42^{\circ} \mathrm{C}$.

\begin{tabular}{|c|c|c|c|c|c|c|c|c|c|c|c|c|c|c|c|}
\hline \multirow{2}{*}{\multicolumn{2}{|c|}{ Dose }} & \multirow{2}{*}{\multicolumn{2}{|c|}{$\begin{array}{l}\text { Température et durée } \\
\text { de conservation }\end{array}$}} & \multicolumn{4}{|c|}{ Culture E } & \multicolumn{4}{|c|}{ Culture 4} & \multicolumn{4}{|c|}{ Culture U } \\
\hline & & & & I & II & III & IV & I & II & III & IV & $\mathbf{I}$ & II & III & IV \\
\hline 2.5 & p. 100 & Cultur & fraîche & $2^{05}$ & $3^{15}$ & $1^{10}$ & & $2^{20}$ & $3^{25}$ & $1^{15}$ & & $2 \mathrm{~h}$ & $3^{15}$ & $1^{15}$ & \\
\hline \multirow[t]{7}{*}{5} & p. 100 & Fraîcheme & t lyophilisée & $2^{05}$ & $4^{55}$ & $2^{50}$ & 0.12 & $2^{10}$ & $5 \mathrm{~h}$ & $2^{50}$ & 0.25 & $2 \mathrm{~h}$ & $5 \mathrm{~h}$ & $3 \mathrm{~h}$ & 0.50 \\
\hline & & $4^{\circ} \mathrm{C}$ & 3 mois & $3^{35}$ & $6^{30}$ & $2^{55}$ & & $2^{45}$ & $5^{25}$ & $2^{40}$ & & $2^{35}$ & $4^{35}$ & $2 \mathrm{~h}$ & \\
\hline & & $4^{\circ} \mathrm{C}$ & 9 mois & $3^{20}$ & $6^{15}$ & $2^{55}$ & 0.20 & $3^{10}$ & $5^{10}$ & $2 \mathrm{~h}$ & 0.30 & $3^{30}$ & $5^{10}$ & $1^{40}$ & 0.50 \\
\hline & & $20-25^{\circ} \mathrm{C}$ & 3 mois & $3^{35}$ & $7^{20}$ & $3^{45}$ & & $3 \mathrm{~h}$ & $5^{25}$ & $2^{25}$ & & $3^{10}$ & $6^{10}$ & $3 \mathrm{~h}$ & \\
\hline & & $20-25^{\circ} \mathrm{C}$ & 9 mois & $5 \mathrm{~h}$ & $8^{40}$ & $3^{40}$ & 0.05 & $5^{05}$ & $7^{45}$ & $2^{40}$ & 0.20 & $5^{10}$ & $7^{50}$ & $2^{40}$ & 0.20 \\
\hline & & $35^{\circ} \mathrm{C}$ & 3 mois & $6 \mathrm{~h}$ & $9^{15}$ & $3^{15}$ & & & & & & $6^{35}$ & $10 \mathrm{~h}$ & $3^{25}$ & \\
\hline & & $35^{\circ} \mathrm{C}$ & 6 mois & $7^{45}$ & $12^{30}$ & $4^{45}$ & 0.02 & $8^{30}$ & $14 \mathrm{~h}$ & $5^{3 n}$ & 0.05 & $7^{30}$ & $13 \mathrm{~h}$ & $5^{30}$ & 0.02 \\
\hline \multirow[t]{5}{*}{2.5} & p. 100 & Fraîcheme & at lyophilisée & $2^{55}$ & $5^{05}$ & $2^{30}$ & 0.20 & $2^{55}$ & $5^{40}$ & $2^{45}$ & 0.50 & $2^{25}$ & $5^{05}$ & $2^{40}$ & 0.50 \\
\hline & & $4^{\circ} \mathrm{C}$ & 9 mois & $3^{50}$ & $6^{10}$ & $2^{20}$ & 0.20 & $3^{40}$ & $5^{25}$ & $1^{45}$ & 0.20 & $3^{45}$ & $5^{30}$ & $1^{45}$ & 0.20 \\
\hline & & $20-25^{\circ} \mathrm{C}$ & 7 semaines & $3^{45}$ & $7^{30}$ & $3^{45}$ & & $3^{55}$ & $6^{30}$ & $2^{35}$ & & $4 \mathrm{~h}$ & $6^{30}$ & $2^{30}$ & \\
\hline & & $20-25^{\circ} \mathrm{C}$ & 9 mois & $6 \mathrm{~h}$ & $8^{30}$ & $2^{30}$ & 0.20 & $5^{25}$ & $7^{65}$ & $1^{40}$ & 0.20 & $5^{35}$ & $8^{10}$ & $2^{35}$ & 0.10 \\
\hline & & $35^{\circ} \mathrm{C}$ & 7 semaines & $5^{40}$ & $8^{35}$ & $2^{55}$ & & $5^{35}$ & $9 \mathrm{~h}$ & $3^{25}$ & & $5^{55}$ & $8^{20}$ & $2^{55}$ & \\
\hline 1.0 & p. 100 & Fraîcheme & at lyophilisée & $3^{40}$ & $6^{30}$ & $2^{50}$ & 0.50 & $3^{10}$ & $6^{25}$ & $2^{45}$ & 0.35 & & & & \\
\hline 0.5 & p. 100 & Fraîcheme & at lyophilisée & $4 \mathrm{~h}$ & $7 \mathrm{~h}$ & $3 \mathrm{~h}$ & 0.50 & $4 \mathrm{~h}$ & $6^{45}$ & $2^{45}$ & 0.35 & & & & \\
\hline 0.25 & p. 100 & Fraîcheme & at lyophilisée & $4^{30}$ & $7^{10}$ & $2^{40}$ & 1.00 & $4^{20}$ & $7^{05}$ & $2^{45}$ & 0.50 & & & & \\
\hline & $I=t$ & ps en heur & pour atteind & $\mathrm{pHc}$ & 5.00 & & & $=\Delta$ & $=\mathrm{II}$ & & & & & & \\
\hline & $\mathrm{II}=\mathrm{t}$ & ps en heur & pour atteind & $\mathrm{pH}$ & 4.30 & & & $=$ in & ice lac & obaci & /stre & oque & à $\mathrm{pH}$ & 4.30 & \\
\hline
\end{tabular}


Ces tableaux, qui nous donnent en plus des renseignements sur la dose d'inoculation et l'influence de la température de conservation des cultures sèches, nous indiquent que les cultures séparées de leur milieu de croissance ont des facultés de conservation légèrement supérieures. Alors qu'à la température de $4^{\circ} \mathrm{C}$, les cultures de lait concentré semblent avoir une conservation un peu meilleure que les cultures resuspendues dans un milieu de lyophilisation, cellesci supportent mieux les températures ambiantes et tropicales, ce qui se traduit par un temps d'acidification un peu plus court, mais surtout par un indice lactobacilles/streptocoques beaucoup plus stable. C'est pourquoi nous pensons que l'emploi de cultures de lait concentré est parfaitement recommandable pour produire des cultures lyophilisées de yoghourt, mais que si ces cultures doivent supporter des conditions de stockage rigoureuses, il faudra leur préférer les cultures séparées de leur milieu de croissance.

Il va de soi que, dans la mesure du possible, il faudra stocker les unes et les autres à la température la plus basse possible. La dose d'inoculation, surtout pour les cultures de lait concentré, ne devra pas excéder 1 p. 100 de culture reconstituée. Des doses inférieures n'ont pas d'effet défavorable sur l'équilibre, bien au contraire, mais rallonge quelque peu le temps d'acidification.

\section{DISCUSSION}

Si la lyophilisation des cultures normales de yoghourt a pour effet de les déséquilibrer en tuant les lactobacilles plus que les streptocoques, il est possible de produire des cultures équilibrées soit en les séparant de leur milieu de croissance, soit en les faisant croître, avant la lyophilisation, dans du lait concentré.

Il semble que l'effet principal de la première méthode repose sur la séparation, avec le milieu de croissance, des peptides et des acides aminés protéolysés par Lactobacillus bulgaricus. Ces subsstances apportées avec la culture lyophilisée stimulent unilatéralement la croissance de streptococcus thermophilus.

La seconde méthode semble exercer une action directe sur Lactobacillus bulgaricus en augmentant sa résistance et en le proté. geant au cours de la lyophilisation. Il n'est pas exclu que dans ce nouveau milieu de croissance, la quantité d'acides aminés libres stimulant la croissance unilatérale de Streptococcus thermophilus soit plus limitée.

Les cultures lyophilisées de yoghourt, bien qu'étant un peu plus lentes que les cultures fraîches, leur sont parfaitement comparables sur le plan des qualités technologiques. En général, lorsque le temps total d'acidification n'excède pas six heures pour les cultures de lait concentré et huit heures pour les cultures séparées de leur milieu de 
croissance, l'examen organoleptique du yoghourt fabriqué avec elles est satisfaisant.

Mais ces cultures, trop onéreuses pour la fabrication directe, sont réservées à la préparation des cultures mères. Or dans ces conditions, l'emploi de cultures même épuisées par un stockage long et rigoureux est parfaitement possible et conduit à un yoghourt de bonne qualité.

\section{$R$ é $s$ u $m$ é}

Les cultures de yoghourt sont composées de deux micro-orga. nismes, Streptococcus thermophilus et Lactobacillus bulgaricus. Leur milieu de croissance naturel est le lait. Si l'on soumet à la lyophilisation une culture normale de yoghourt, on provoque en son sein un déséquilibre bactériologique, le lactobacille étant plus sensible à ce traitement que le streptocoque. La lyophilisation des cultures pures (Tabl. 1) donne un ordre de grandeur de cette sensibilité. Pour parvenir à maintenir l'équilibre, il est nécessaire de rendre le milieu de lyophilisation (lait) plus protecteur en lui ajoutant soit du lactose ou du saccharose, soit de la poudre de lait écrémé. Mais le procédé consistant à faire croître la culture directement dans un milieu protecteur (lait écrémé concentré) donne des résultats supérieurs.

Le stockage des cultures pendant de longs mois les affaiblit. Cet affaiblissement, fonction de la température, se manifeste à nouveau par un défaut de lactobacilles. Il est possible de lutter contre ce déséquilibre en séparant par centrifugation les bactéries de leur milieu de croissance et en les resuspendant dans un milieu de lyophilisation protecteur. L'absence des acides aminés nécessaires à la croissance de Streptococcus thermophilus gêne son développement et permet à Lactobacillus bulgaricus de combler son retard.

\section{S u m m a ry}

Yoghurt cultures consist of Str. thermophilus and Lb. bulgaricus. Their natural medium is milk. Freeze-drying of normal yoghurt cultures causes a disturbance of the équilibrium, because $L b$. bulgaricus suffers more from this treatment than Str. thermophilus.

Freeze-drying of both strains separately (tabl. 1) shows how delicate these microorganisms are. In order to maintain the equilibrium of the mixed culture, it is necessary to increase the protective effect of the medium milk by adding either lactose, or saccharose, or skimmilk powder. It is even more helpful to have the cultures grow in this improved medium from the beginning. 
The activity of the cultures is decreasing after storage of several months. Specially the lactobacilli are more impaired at higher temperatures. This can be avoided by separating the organism from the medium and putting them into a fresh protective suspension.

In this case the early development of Str. thermophilus is inhibited by the absence of amino acids necessary for its growth. This gives $L b$. bulgaricus more time to develop.

\section{Bibliographie}

Сно (С.). and ОвауаSнi (Y.) (1956). - "Effect of adjuvant on preservability of dried B.C.G. vaccine at $37^{\circ} \mathrm{C}$ ». Bull. Wld. Hlth. Org. 14, 657.

Cho (C.)., ObaYashi (Y.), Ywasaki (T.) and KaWASAKi (J.) (1956). " Effect of storage at $37^{\circ} \mathrm{C}$ on immunizing power of dried B.C.G. vaccine ». Bull. Wld. Hlth. Org. 14, 671.

FRY (R.M.). (1951). - "The influence of the suspending fluid on the survival of bacteria after drying ». Freezing and Drying, p. 107, Institute of Biology, London.

FRY (R.M.). and GREAVES (R.I.N.). (1951). - " The survival of bacteria before and after drying». J. Hyg. (Camb.), 49, 220-246.

GaviN (M.). (1968 b). - « La lyophilisation des cultures de yoghourt ». Thèse No 4227 de l'Ecole Polytechnique Fédérale, Zürich.

Nikolov (N.). (1964). - "Study on the stability of freeze-dried yoghurt cultures". Zhivotn. Nauki, Sofia, 1, 93-98. Réf. DSA 26 (1964), 3486.

Pette (J.W.) and Lolkema (I.H.) (1950). - " Symbiosis and Antibiosis in mixed Cultures of Lb. bulgaricus and Sc. thermophilus ». Neth. Milk and Dairy J., 4, 197-208.

Scotr (W.J.) (1958). - " The effect of residual water on the survival of dried bacteria during storage ". J. gen. Microbiol. 19, 624-633.

Reçu pour publication en décembre 1968. 